\title{
Overpowering posterior lumbar instrumentation and fusion with hyperlordotic anterior lumbar interbody cages followed by posterior revision: a preliminary feasibility study
}

\author{
Abhijeet Kadam, MD, Nathan Wigner, MD, PhD, Philip Saville, MD, and Vincent Arlet, MD \\ Department of Orthopaedic Surgery, Penn Medicine, University of Pennsylvania, Philadelphia, Pennsylvania
}

OBJECTIVE The authors' aim in this study was to evaluate whether sagittal plane correction can be obtained from the front by overpowering previous posterior instrumentation and/or fusion with hyperlordotic anterior lumbar interbody fusion (ALIF) cages in patients undergoing revision surgery for degenerative spinal conditions and/or spinal deformities.

METHODS The authors report their experience with the application of hyperlordotic cages at 36 lumbar levels for ALIFs in a series of 20 patients who underwent revision spinal surgery at a single institution. Included patients underwent staged front-back procedures: ALIFs with hyperlordotic cages $\left(12^{\circ}, 20^{\circ}\right.$, and $\left.30^{\circ}\right)$ followed by removal of posterior instrumentation and reinstrumentation from the back. Patients were divided into the following 2 groups depending on the extent of posterior instrumentation and fusion during the second stage: long constructs ( $\geq 6$ levels with extension into thoracic spine and/or pelvis) and short constructs ( $<6$ levels). Preoperative and postoperative standing radiographs were evaluated to measure segmental lordosis (SL) along with standard sagittal parameters. Radiographic signs of pseudarthrosis at previously fused levels were also sought in all patients.

RESULTS The average patient age was 54 years (range 30-66 years). The mean follow-up was 11.5 months (range 5-26 months). The mean SL achieved with $12^{\circ}, 20^{\circ}$, and $30^{\circ}$ cages was $13.1^{\circ}, 19^{\circ}$, and $22.4^{\circ}$, respectively. The increase in postoperative $S \mathrm{~L}$ at the respective surgically treated levels for $12^{\circ}, 20^{\circ}$, and $30^{\circ}$ cages that were used to overpower posterior instrumentation/fusion averaged $6.1^{\circ}(p<0.05), 12.5^{\circ}(p<0.05)$, and $17.7^{\circ}(p<0.05)$, respectively. No statistically significant difference was found in SL correction at levels in patients who had pseudarthrosis $(n=18)$ versus those who did not $(n=18)$. The mean overall lumbar lordosis increased from $44.3^{\circ}$ to $59.8^{\circ}(p<0.05)$. In the long-construct group, the mean improvement in sagittal vertical axis was $85.5 \mathrm{~mm}$ (range $19-249.3 \mathrm{~mm}, \mathrm{p}<0.05$ ). Endplate impaction/ collapse was noted in 3 of 36 levels (8.3\%). The anterior complication rate was $13.3 \%$. No neurological complications or vascular injuries were observed.

CONCLUSIONS ALIF in which hyperlordotic cages are used to overpower posterior spinal instrumentation and fusion can be expected to produce an increase in SL of a magnitude that is roughly half of the in-built cage lordotic angle. This technique may be particularly suited for lordosis correction from the front at lumbar levels that have pseudarthrosis from the previous posterior spinal fusion. Meticulous selection of levels for ALIF is crucial for safely and effectively performing this technique.

https://thejns.org/doi/abs/10.3171/2017.5.SPINE16926

KEY WORDS overpowering posterior fusion; ALIF; hyperlordotic cage; pseudarthrosis; lumbar; deformity

$\mathrm{T}$ HE importance of the sagittal plane in adult spine deformity is well established. Failure to restore lumbar lordosis (LL) following fusion not only accelerates adjacent-segment degeneration ${ }^{14,20}$ but also has an adverse effect on a patient's symptomatology, qual- ity of life measures, and overall function. ${ }^{17,18}$ With an expected increase in the rates of revision spine surgery in the future, ${ }^{13}$ lumbar hypolordosis in the presence of previous posterior instrumentation and/or fusion will likely be a growing problem. Conventional strategies for lumbar

ABBREVIATIONS ACR = anterior column realignment; $\mathrm{ALIF}=$ anterior lumbar interbody fusion; $\mathrm{ALL}=$ anterior longitudinal ligament; $\mathrm{AP}=$ anteroposterior; $\mathrm{BMI}=$ body mass index; $\mathrm{LL}=$ lumbar lordosis; $\mathrm{PI}=$ pelvic incidence; $\mathrm{PLL}=$ posterior longitudinal ligament; $\mathrm{PT}=$ pelvic tilt; $\mathrm{SL}=$ segmental lordosis; $\mathrm{SS}=$ sacral slope; $\mathrm{SVA}=$ sagittal vertical axis; TCO = 3-column osteotomy; TLIF = transforaminal lumbar interbody fusion.

SUBMITTED August 4, 2016. ACCEPTED May 1, 2017.

INCLUDE WHEN CITING Published online September 29, 2017; DOI: 10.3171/2017.5.SPINE16926. 
flat-back deformity involve addressing sagittal malalignment from the back with Smith-Petersen osteotomy and 3-column osteotomies (TCOs), including pedicle subtraction osteotomy and, to a lesser extent, vertebral column resection. Although they are powerful realignment techniques, TCOs can be demanding to perform in revision scenarios due to scar tissue. ${ }^{2}$ In addition, high rates of overall complications ${ }^{3,5,8}$ (up to $58 \%{ }^{12}$ ), including significant neurological complication rates, ${ }^{6}$ have been reported for TCOs. These rates are likely to be even higher in revision surgeries. Another strategy for flat-back correction is the $540^{\circ}$ fusion procedure (back-front-back), a procedure classically described before the advent of posterior TCOs. This procedure consists of removal of posterior instrumentation, posterior release/osteotomies and reinsertion of pedicle screws, and closing the posterior wound in the first stage. The second stage includes turning the patient supine and performing anterior lumbar interbody fusion (ALIF). The third stage includes reopening the posterior wound and inserting rods to maintain correction. The cumbersome nature of the procedure, involving multiple flips and patient repositioning, as well as prolonged surgical time are inherent drawbacks of the $540^{\circ}$ fusion procedure. The technique of "overpowering" posterior spinal instrumentation, as presented in this paper, eliminates an entire stage of the traditionally described $540^{\circ}$ procedure.

Recent work published on anterior column realignment (ACR) has renewed the interest in anterior-based correction strategies for sagittal plane deformity. $1,4,7,9,11$, ${ }^{15,19}$ Release of the anterior longitudinal ligament (ALL) and the use of hyperlordotic interbody cages to achieve lengthening and fusion of the anterior column of the spine are important principles underlying ACR. However, ACR is conventionally performed using minimally invasive lateral transpsoas- or anterolateral-based approaches, which have limited utility for the more severe spectrum of sagittal plane deformity cases. ${ }^{10}$ In addition, the presence of previous posterior/instrumentation and/or fusion (as is frequently encountered in revision cases) precludes alignment correction and release with minimally invasive approaches (at the posteriorly fused levels), without prior removal of instrumentation. We believe that with the versatility of the open retroperitoneal approach, the principles of ACR can be applied for correction in the lumbar spine from the front, even in the presence of intact previous posterior instrumentation or fusion. Also, as discussed before, the need for the 3 -staged $540^{\circ}$ fusion procedure may be obviated with the novel technique of overpowering the posterior lumbar instrumentation and/or fusion with anterior interbody cages, as presented in this paper.

\section{Methods}

\section{Patient Population}

After obtaining IRB approval, we performed a retrospective review and radiographic analysis of consecutive adult patients who had undergone surgery performed by a single surgeon at a single institution from November 2014 to April 2016. Patients with degenerative spinal conditions and/or spinal deformities who underwent ALIFs at levels that were previously fused posteriorly formed the target study population. The ALIF procedures were performed as a part of staged anterior and posterior fusion.

Inclusion criteria were 1) age older than 18 years, and 2) ALIF with insertion of 1 or more hyperlordotic cages at levels that had previously been treated with posterior instrumentation/fusion. Exclusion criteria were 1) inadequate pre- or postoperative imaging, 2) osteoporosis (hip T-score $<-1.5)$ and body mass index $(\mathrm{BMI})>40$. For the purpose of this study, ALIF cages with $12^{\circ}, 20^{\circ}$, and $30^{\circ}$ of built-in lordosis manufactured by 2 companies were used: Brigade polyetheretherketone cages $\left(12^{\circ}, 20^{\circ}\right.$, and $30^{\circ}$; NuVasive Inc.), and Cougar carbon fiber reinforced polymer cages $\left(20^{\circ}\right.$; DePuy Synthes Spine Inc.). The choice of the cages to be inserted was determined preoperatively by analyzing the spinopelvic parameters with the aim of restoring LL as close to the pelvic incidence (PI) as possible.

\section{Surgical Technique}

An anterior retroperitoneal approach was used for all ALIFs. All approaches were done with the assistance of an access surgeon and with the patient in the supine position. The borders of the disc space were clearly identified, and the ALL was divided into 2 flaps in an H-shaped configuration. The disc was then incised and separated from the inferior and superior endplates with the aid of Cobb elevators and rongeurs. If the disc space was obscured by osteophytes or a previous posterior transforaminal lumbar interbody fusion (TLIF), an anterior osteotomy was performed with the aid of medium-sized osteotomes to facilitate access to the disc space. In cases of previous TLIF fusion, a narrow disc distractor was inserted adjacent to the spacer and progressively distracted. Curettage around the TLIF spacer was done to delineate the margins of the TLIF implant and to separate it from the endplates and/or posterior longitudinal ligament (PLL). Care was taken to preserve the endplates to prevent subsidence of the anterior cages.

The following stepwise approach was used to sequentially distract the disc space and overpower posterior instrumentation and fusion for obtaining desired lordosis at the surgically treated level. 1) Extensive exposure of the disc space was made so that a very wide discectomy/release could be performed. 2) Complete release of the ALL and extensive discectomy up to the PLL was performed, with release of the PLL in most cases (except at levels with a previous TLIF). 3) A large paddle distractor was inserted after the discectomy release to pry open the disc space. To give more power to the distractor, a small 8/10-mm TLIF paddle was inserted between the blades of the distractor and rotated $90^{\circ}$ in place to pry open the disc space by overpowering the posterior instrumentation/fusion. The distractor was inserted as far back to the posterior rim of the endplate as possible in order to avoid breaking the endplate during distraction. 4) The jackknife capability of the operating room table provided gradual, controlled generation of lordosis. The patient's lumbosacral junction was positioned at the level of the hinge of the table, and during the discectomy the table was "jackknifed" into further lordosis. 5) Hyperlordotic templates were then inserted sequentially from smallest to largest size to open up the disc space further before the final cage was inserted.

A hyperlordotic ALIF cage packed with osteoconduc- 
tive material (Osteocel Plus, NuVasive Inc.) soaked in bone marrow aspirate was then inserted into the prepared disc space. Depending on the manufacturer's design, locking screws were placed into the endplates through the cage, or cortical screws with a washer were placed into the inferior endplate adjacent to the cage. Screw fixation was essential, as the natural tendency of these lordotic spacers is to extrude. As posterior instrumentation was planned, screws were placed into 1 endplate at most of the levels to allow for further lordosis correction from the back, if needed. Thus, further correction posteriorly could potentially be achieved by a combination of release of posterior elements with facetectomies and slightly overcontouring and in situ bending of the rods at the time of posterior instrumentation and fusion. Anteroposterior (AP) and lateral fluoroscopic images were obtained to check the desired positioning of the cage and whether good endplate-to-endplate contact was obtained.

The posterior instrumentation and posterior spinal fusion procedures were performed 0-7 days after the anterior procedure. Spinal levels instrumented in the posterior fusion varied depending on the pathology being addressed.

\section{Radiographic Analysis}

Digital standard upright lateral radiographs were used to measure sagittal parameters on all patients' pre- and postoperative images: LL, defined as the angle between the L-1 superior endplate to the S-1 superior endplate; segmental lordosis (SL) at ALIF levels, defined as the angle between the superior and inferior endplates of a disc space; pelvic tilt (PT); PI; and sacral slope (SS). An independent, nonblinded observer recorded all measurements. Each set of measurements was done twice for consistency.

\section{Statistical Analysis}

A 2-sample paired t-test for means was used to compare preoperative and postoperative values for the different spinopelvic parameters measured. Statistical significance was set at $p<0.05$. Depending on the extent of the posterior fusion that was performed in the second stage, patients were categorized into one of 2 groups: the shortconstruct group (defined as posterior spinal instrumentation limited to only the lumbar spine segments, i.e., $<6$ levels) and the long-construct group (defined as posterior spinal instrumentation extending either into the thoracic spine proximally or the pelvis distally, i.e., $\geq 6$ levels). Analysis of pre- and postoperative spinopelvic parameters was performed, both overall and separately for these 2 groups. Furthermore, the levels of ALIFs were divided based on the presence or absence of pseudarthrosis from the previous posterior fusion (as evident on preoperative imaging). Segmental lordosis was compared for ALIFs in these 2 groups separately as well.

\section{Results \\ Demographics}

A total of 20 patients ( 12 men and 8 women) met the inclusion criteria, with a total of 36 implanted cages at levels that had been treated with previous posterior instrumentation or fusion. The mean patient age was 54 years (range

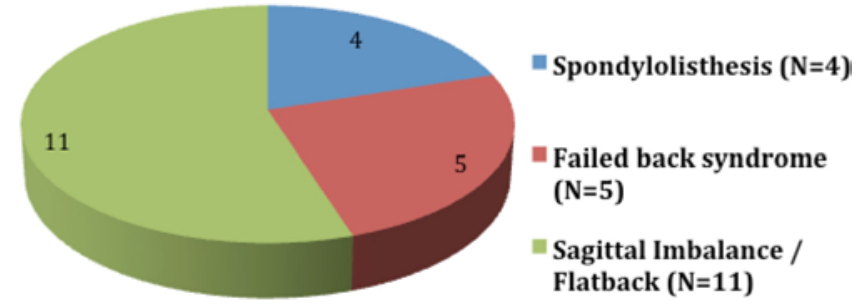

FIG. 1. Surgical indications. Figure is available in color online only.

30-66 years). The mean BMI of the patients in the study was 29.0 (range 21.13-38.62). The mean duration of radiographic follow-up in our series up was 11.5 months (range 5-26 months). The breakdown of the primary surgical indications is given in Fig. 1, and Table 1 summarizes the demographic data for our series. A few illustrative cases from the series are described in Figs. 2-10.

\section{Levels of ALIFs and Cages Implanted}

The cages used for the ALIFs and the levels of posterior instrumentation/fusion that were overpowered are shown in Table 2 . The majority of cages were implanted at the L5-S1 level $(n=15)$, followed by the L4-5 level ( $=10$ ). Likewise, most of the cages implanted were of $30^{\circ}$ lordotic profile $(n=21)$. Only 2 cages of the 36 implanted levels were carbon fiber-reinforced polymer cages $\left(20^{\circ}\right.$; Cougar). The vast majority were polyetheretherketone cages $\left(12^{\circ}, 20^{\circ}\right.$, and $30^{\circ}$; Brigade).

\section{Levels of Posterior Instrumentation and Fusion Done in Second Stage}

Eleven of 20 patients had long posterior-instrumented fusions ( $\geq 6$ levels, with fusion extending to the thoracic spine proximally and/or to the pelvis distally).

\section{Characteristics of the Previous Posterior Instrumentation/ Fusion}

Pseudarthrosis from previous posterior instrumenta-

TABLE 1. Demographic data

\begin{tabular}{cc}
\hline \multicolumn{1}{c}{ Variable } & Value \\
\hline No. of patients & 20 \\
\hline No. of ALIF cages placed & 36 \\
\hline Sex, no. & 12 \\
\hline M & 8 \\
\hline F & \\
\hline Age, yrs & $54(9.7)$ \\
\hline Mean (SD) & $30-66$ \\
\hline Range & \\
\hline BMl & $29.0(4.9)$ \\
\hline Mean (SD) & $21.1-38.6$ \\
\hline Range & $11.5(4.8)$ \\
\hline Follow-up, mos & $5-26$ \\
\hline Mean (SD)
\end{tabular}



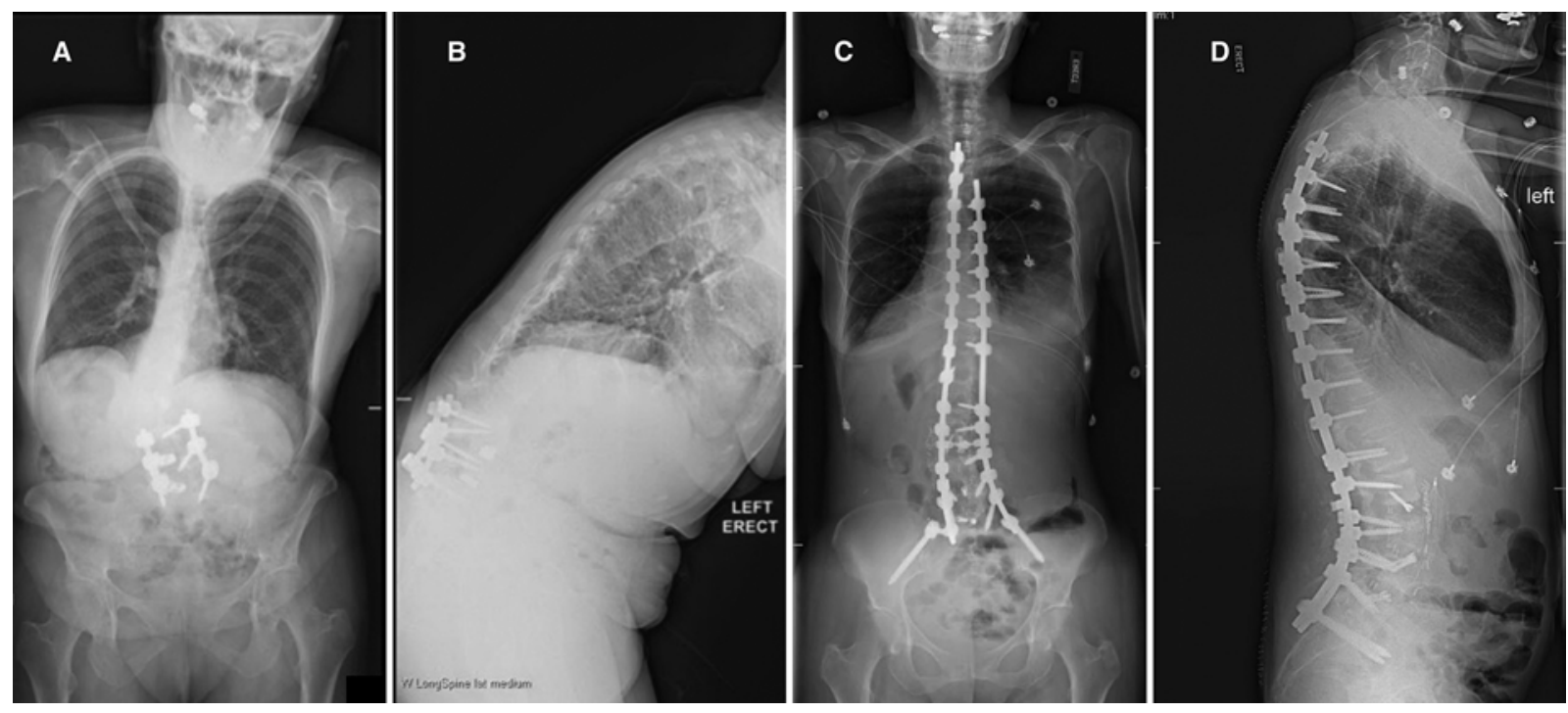

FIG. 2. A and B: Preoperative full-length standing AP $(A)$ and lateral $(B)$ radiographs obtained in a 58-year-old patient with severe sagittal and coronal plane imbalance. Note the previous L3 -5 posterior instrumentation and TLIFs. L-5 is sacralized in this patient. C and D: Postoperative full-length radiographs showing correction of the SVA to $60 \mathrm{~mm}$. Lumbar lordosis corrected to $70^{\circ}$ with removal of the TLIF cages at L3-4 and L4-5 and overpowering these levels with $12^{\circ}$ and $30^{\circ}$ cages. In addition, another $30^{\circ}$ cage was implanted at L2-3.

tion/fusion was documented at 18 of the 36 levels treated with ALIF. A detailed breakdown is provided in Table 3. Two patients in the short-construct group had previously undergone TLIFs at 3 levels (2 at L5-S1 and 1 at L3-4), which were not solid. Screw breakage was observed in one of these patients at the L3-4 level. No rod failures were seen in the short-construct group. In the long-construct group, 4 patients had previously undergone TLIF (6 levels total: 2 patients with 1 level each and 1 patient with 2 levels). Rod failures were present in 2 patients in the longconstruct group, one of which occurred in a patient who had a previous TLIF at the level of rod breakage.

One patient in the long-construct group had posterior instrumentation removed before presenting to us and had solid posterolateral fusion, which was overpowered with a 1-level ALIF. The patient underwent an L1-pelvis fusion in the second stage for correction of her flat-back deformity.

All patients in our series had previous posterior instrumentation with titanium $(n=14)$ or stainless steel $(n=6)$ rod construct systems; none had cobalt chrome rods. The retrieved rods were from 5.5-, 6.0-, and 6.35-mm-diameter systems. Both cases of rod failure in the long-construct group (described above) had 6.35-mm stainless steel rod systems from their previous surgery.

\section{Spinopelvic Sagittal Parameters}

A detailed analysis of the change in the overall LL, PT, SS, and PI-LL mismatch is provided in Table 4.

\section{Long-Construct Group}

The-mean PI for patients in the long-construct group
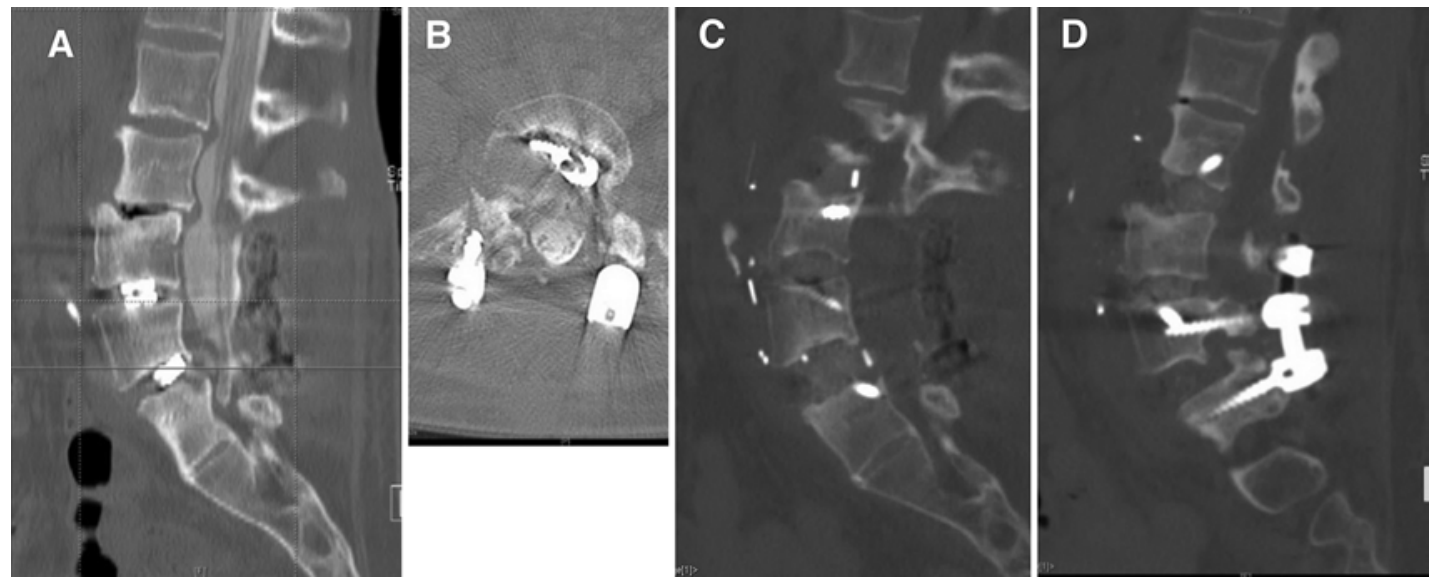

FIG. 3. A and B: Preoperative sagittal (A) and axial (B) CT scans obtained in the patient shown in Fig. 2, demonstrating vacuum signs at $L 4-5$ and $L 2-3$, with lucency around the cage at $L 4-5$ suggestive of pseudarthrosis. C and D: Postoperative sagittal CT scans obtained after the front stage of the procedure, demonstrating good alignment correction with the ALIF cages. 

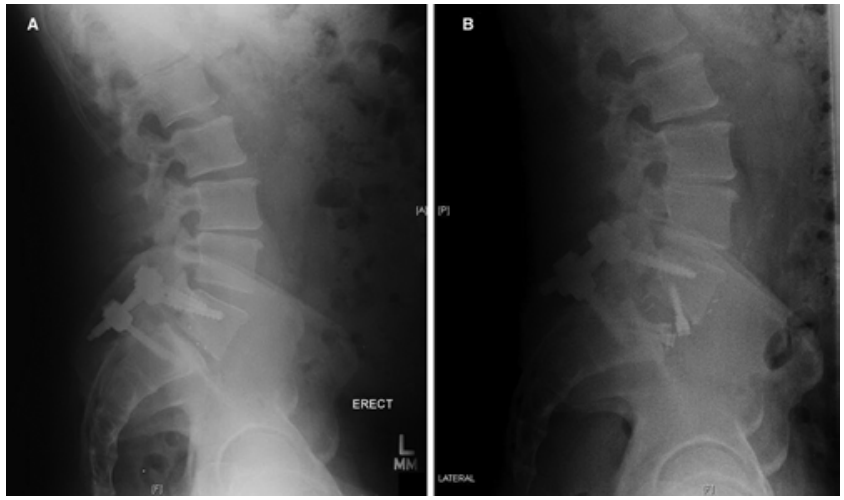

FIG. 4. A: Preoperative erect lateral lumbar radiograph obtained in a 57-year-old man with spondylolisthesis and previous L5-S1 posterior instrumentation and fusion with TLIF. Note the increased opening up of disc spaces proximal to the fusion to compensate for loss of lordosis at L5-S1. B: Postoperative erect lateral radiograph showing good correction with the $30^{\circ}$ ALIF cage used to overpower the posterior instrumentation after removal of the TLIF cage. Segmental lordosis corrected from $10^{\circ}$ to $28^{\circ}$. Disc spaces above the fusion have fallen back to the normal range of intersegmental lordosis.

was $67.8^{\circ}\left(\mathrm{SD} 7.3^{\circ}\right.$; range $\left.57.4^{\circ}-79.7^{\circ}\right)$. The mean LL increased from a preoperative value of $35.6^{\circ}$ to $57.5^{\circ}$ postoperatively $(\mathrm{p}<0.05)$. Similarly, the PI-LL mismatch in this group decreased significantly from a mean of $32.2^{\circ}$ to $10.3^{\circ}(\mathrm{p}<0.05)$. SS and PT measurements also showed statistically significant improvement.

\section{Short-Construct Group}

The mean PI for patients in the short-construct group was $58.7^{\circ}$ (SD $12.7^{\circ}$; range $\left.44.8^{\circ}-77.4^{\circ}\right)$. All spinopelvic sagittal parameters in this group of patients improved, with the LL increasing postoperatively from a mean of $55.0^{\circ}$ (range $38^{\circ}-75^{\circ}$ ) to $62.7^{\circ}$ (range $52.2^{\circ}-76^{\circ}$ ) (p < $0.05)$. The PI-LL mismatch decreased from a mean of $3.5^{\circ}$
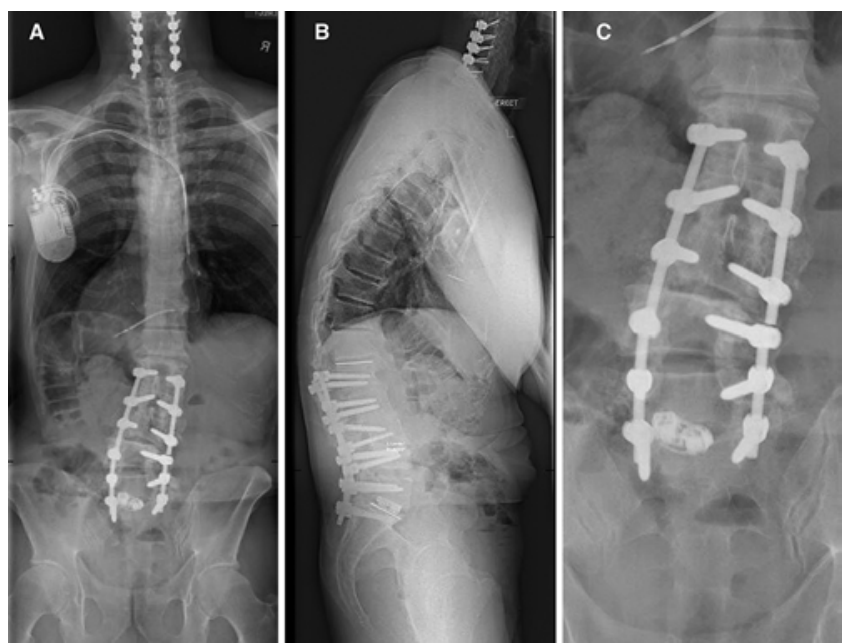

FIG. 5. A and B: Full-length standing $A P(A)$ and lateral $(B)$ radiographs obtained in a 60 -year-old patient with coronal and sagittal imbalance. C: Magnified view of the AP radiograph showing rod breakage at L3-4 and TLIF cage at L5-S1. The preoperative LL was $32^{\circ}$ with an SVA of $75.8 \mathrm{~mm}$. (range $-7.8^{\circ}$ to $15.8^{\circ}$ ) to $-3.9^{\circ}$ (range $-18^{\circ}$ to $\left.13^{\circ}\right)$ (p < $0.05)$. However, the improvements in the SS and PT parameters did not attain statistical significance $(\mathrm{p}>0.05)$.

\section{Segmental Lordosis}

The average amount of absolute SL achieved with $12^{\circ}$, $20^{\circ}$, and $30^{\circ}$ cages was found to be $13.1^{\circ}, 19^{\circ}$, and $22.4^{\circ}$, respectively. The difference in the preoperative and postoperative SL achieved at respective levels with $12^{\circ}, 20^{\circ}$, and $30^{\circ}$ cages was also calculated separately. The mean increase in SL $(\Delta \mathrm{SL})$ produced by $12^{\circ}, 20^{\circ}$, and $30^{\circ} \mathrm{ALIF}$ cages used to overpower posterior instrumentation/fusion was $6.1^{\circ}(\mathrm{p}<0.05), 12.5^{\circ}(\mathrm{p}<0.05)$, and $17.7^{\circ}(\mathrm{p}<0.05)$, respectively (Table 5). Thus, all ALIF cages used were effective in overpowering previous posterior instrumentation and/or fusion at the respective level to recreate SL. Furthermore, for any given degree of ALIF cage used, the gain in SL was found to be roughly half of the built-in lordosis of the cage, at each level. There was no significant difference noted in the increase in SL at levels with pseudarthrosis versus those without (Table 6).

\section{Sagittal Balance}

The mean preoperative sagittal balance as calculated by the sagittal vertical axis (SVA) in the long-construct group was $155 \mathrm{~mm}$ (SD $102.3 \mathrm{~mm}$; range 75.8-318.2 mm). Postoperatively, this improved to $69.5 \mathrm{~mm}$ (SD $25.5 \mathrm{~mm}$; range $25.1-123.2 \mathrm{~mm})(\mathrm{p}<0.05)$. Thus, the SVA improved by an average of $85.5 \mathrm{~mm}$ (SD $89.4 \mathrm{~mm}$; range 19-249.3 $\mathrm{mm}$ ) in this patient group.

\section{Blood Loss}

The average blood loss per patient was $181.2 \mathrm{ml}$ (range 25-400 ml) for anterior surgery alone. Per ALIF, this translated to an estimated average loss of $100.6 \mathrm{ml}$. The average blood loss for posterior staged procedures in our series was $917.5 \mathrm{ml}$ (range 150-2500 ml).

\section{Complications}

All complications observed are listed in detail in Table 7. The complication rate of the anterior surgery was about $13.3 \%$, which included 3 of the $36(8.3 \%)$ ALIF levels showing endplate impaction/collapse intraoperatively. No neurological complications or vascular injuries were observed in our series. There were no cases of retrograde ejaculation in males postoperatively in our series. We observed 1 case of proximal junction kyphosis $(5 \%)$ in the long-construct group.

\section{Loss of Correction}

We defined loss of correction as loss of $>3^{\circ}$ of SL over the follow-up period. A total of 3 cages (8.3\%) displayed loss of SL during follow-up. These were different from the cages in which endplate impaction was observed intraoperatively.

\section{Discussion}

Aside from the established benefits of interbody support and increased fusion efficacy, the use of hyperlordotic 

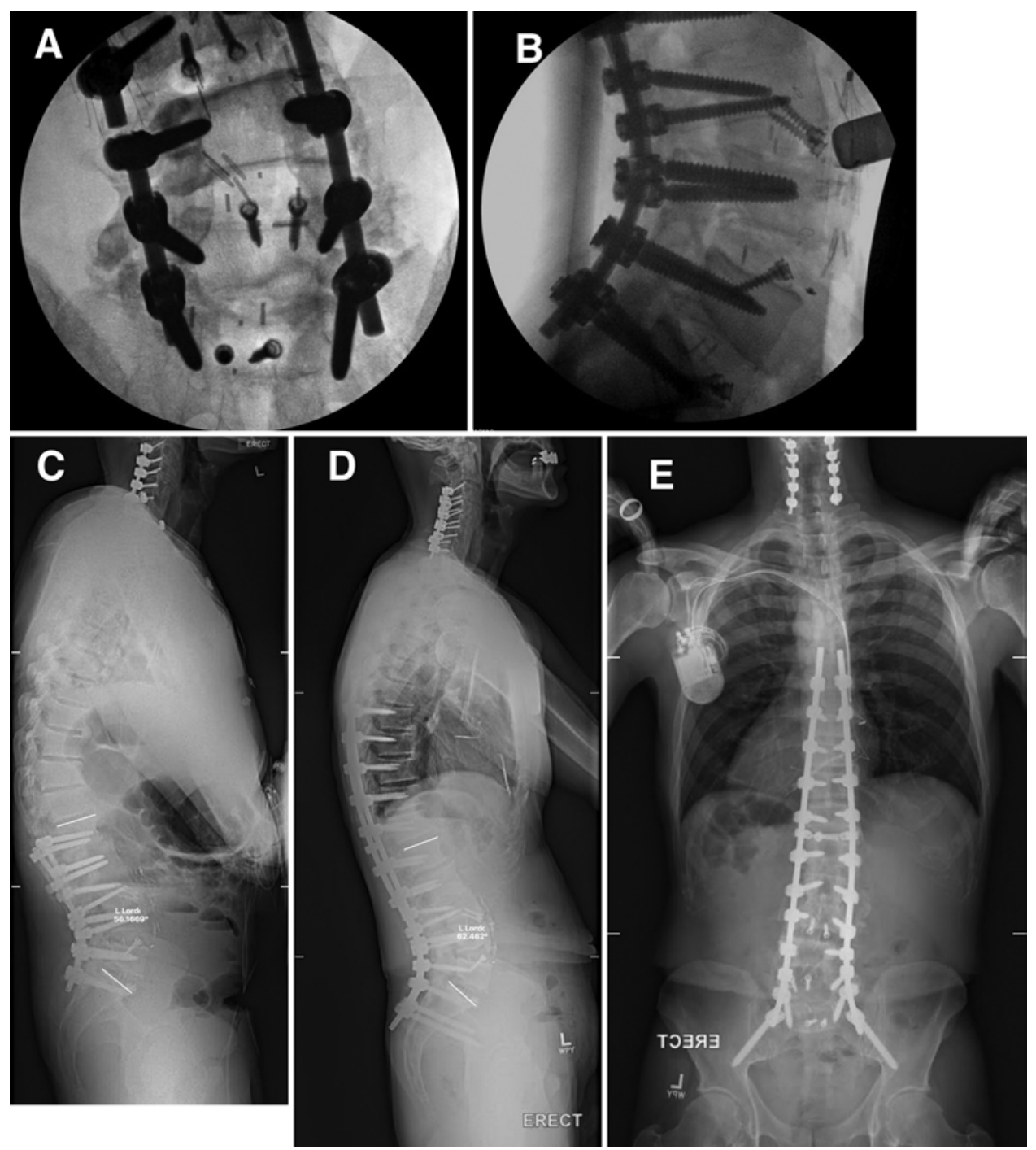

FIG. 6. A and B: Intraoperative fluoroscopy images obtained in the same patient shown in Fig. 5 after insertion of $30^{\circ}, 20^{\circ}$, and $20^{\circ}$ ALIF cages at L5-S1, L4-5, and L3-4, respectively. The posterior instrumentation was overpowered at these 3 levels. The TLIF cage at L5-S1 was found to be loose intraoperatively and was easily retrieved. Note that the endplate screws are placed in only 1 contact endplate per cage to allow for further lordosis correction during the staged posterior procedure. C: Erect standing lateral radiograph obtained before the back procedure, showing lordosis correction to $58^{\circ}$. The patient had a delayed posterior staged procedure in view of medical comorbidities, allowing a radiograph in between the 2 stages to be obtained. D and E: Final postoperative erect lateral and AP radiographs. Notice the final LL increased to $62^{\circ}$, as some minor correction was obtained with simple posterior releases and reinstrumentation. The final SVA is within $25 \mathrm{~mm}$.

ALIF cages has made it possible to correct LL from the front. In a recently published series ${ }^{16}$ we demonstrated that hyperlordotic cages could reliably produce nearly the same degree of SL as is built in the cage at levels without preexisting spondylolisthesis. This is the first such study showing the application of hyperlordotic ALIF cages for alignment correction performed from the front in primary lumbar spine fusion. Building on our experience with the hyperlordotic cages, we could again successfully demonstrate their application in revision lumbar spine cases as well.

In the present study, hyperlordotic cages, used to overpower previous posterior instrumentation and fusion, were shown to produce an increase in SL, amounting to roughly half the built-in lordosis angle of the cage used. When applied at multiple levels, this overpowering ALIF technique supplemented by simple posterior release was found to produce adequate lordosis correction to restore lumbar sagittal alignment. Thus, posterior TCOs with their associated risks and complications of operating in the scar tissue were avoided in our series.

The concept of overpowering posterior instrumentation naturally provokes concerns pertaining to the safety and feasibility of the technique. Vertebral body endplate collapse/fracture, pedicle screw cutout/breakage, and rod failures are some of the intraoperative complications that one might believe are likely to arise while overpowering posterior instrumentation. However, we were able to demonstrate that, with meticulous disc space preparation and a careful sequential distraction technique, the afore- 

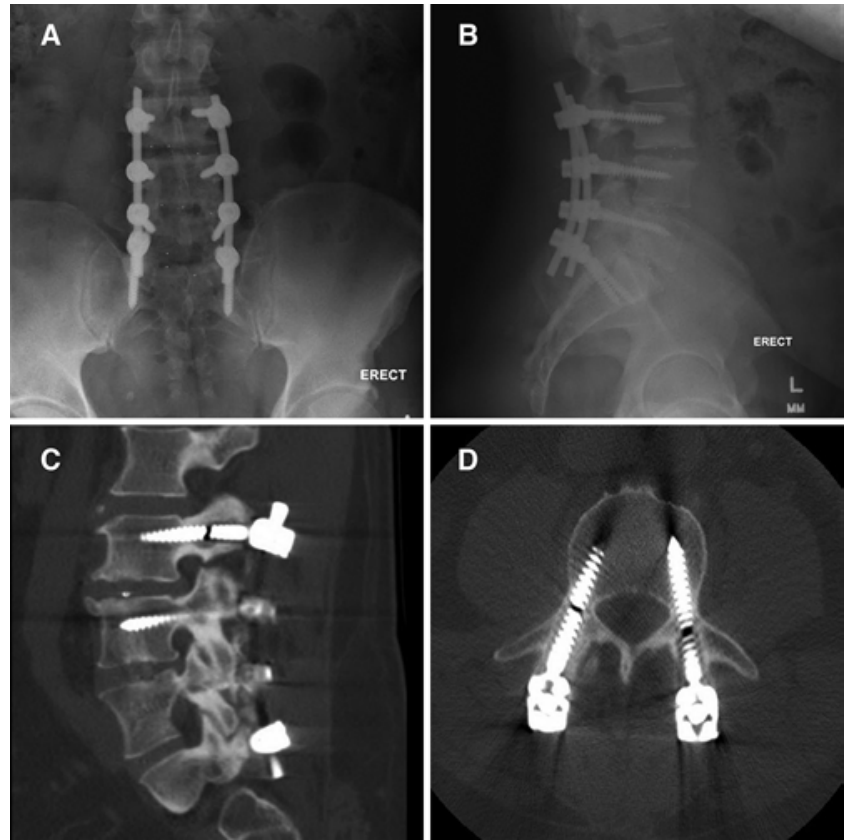

FIG. 7. A and B: AP (A) and lateral $(B)$ erect radiographs obtained in a 48-year-old man with failed-back surgery syndrome and previous TLIFs at $L 3-4, L 4-5$, and L5-S1. The measured LL is $58^{\circ}$. C and D: Note the broken screws in L-3 on preoperative sagittal (C) and axial (D) CT scans, suggestive of pseudarthrosis.

mentioned complications can be avoided. An aggressive discectomy following total release of the ALL, the use of a large footprint endplate distractor with the blades positioned as far posteriorly in the disc space as possible, supplemented by a small TLIF paddle to provide more power to "crack" open the PLL, and the jackknife capability of the operating table to help open up the disc space are all essential steps that we believe are crucial in safely and effectively achieving segmental correction using this technique. In our series of 20 patients with 36 overpowered levels, we noticed endplate impaction at 3 levels. This, in our opinion, resulted from failure to distract the endplates adequately by not positioning the distractor blades as far posteriorly near to the PLL as possible. No intraoperative pedicle screw cutouts or pedicle/vertebral body fractures were noted during or following the overpowering ALIF procedures.

As is true with all complex revision spine surgeries, we were meticulous about patient selection and the levels chosen to be overpowered in order to increase the safety profile of the procedure. Good bone quality is an essential prerequisite for safely overpowering the fused level without causing endplate damage. All included patients were screened with DEXA (dual energy x-ray absorptiometry) scans, and none had osteoporosis (DEXA scan hips: Tscore < -1.5). Moreover, patients with a very high BMI (> 40) were excluded, as safely approaching the deeply situated disc levels and overpowering them in obese patients is a concern. As can be seen from the demographic results, the average BMI in our series was 29 (4.9 SD). The majority of our patients had sagittal imbalance from previous posterior instrumented fusions, an indication that we believe is most appropriately suited for application of our
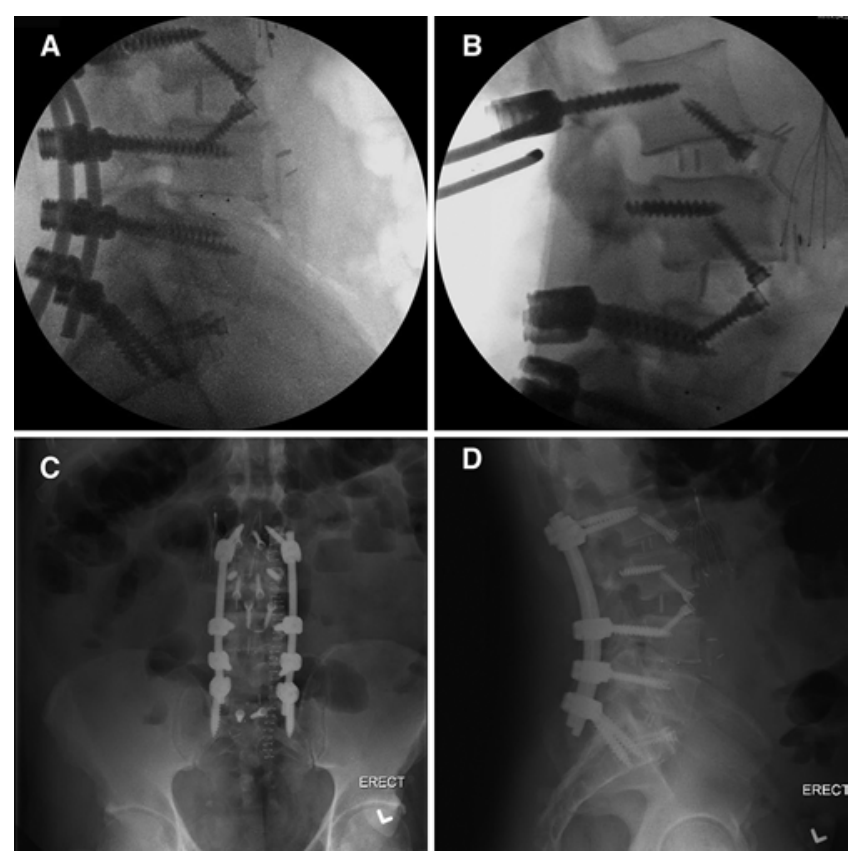

FIG. 8. A: Intraoperative fluoroscopy images (A and B) obtained in the patient shown in Fig. 7 after removal of the TLIF spacers at L3-4 and L5-S1 and insertion of $12^{\circ}$ and $20^{\circ} \mathrm{ALIF}$ cages at these levels, respectively, by the overpowering technique. The fusion at L4-5 was explored and was found to be solid. In addition, another $12^{\circ}$ ALIF cage was inserted at the L2-3 level, which is seen in panel B. B: Removal of posterior instrumentation during the staged back procedure, which was done on the same day. Notice the broken screws in L-3; no attempt was made to take them out. $C$ and D: Final postoperative $A P(C)$ and lateral (D) standing radiographs. Lumbar lordosis increased to $68^{\circ}$.
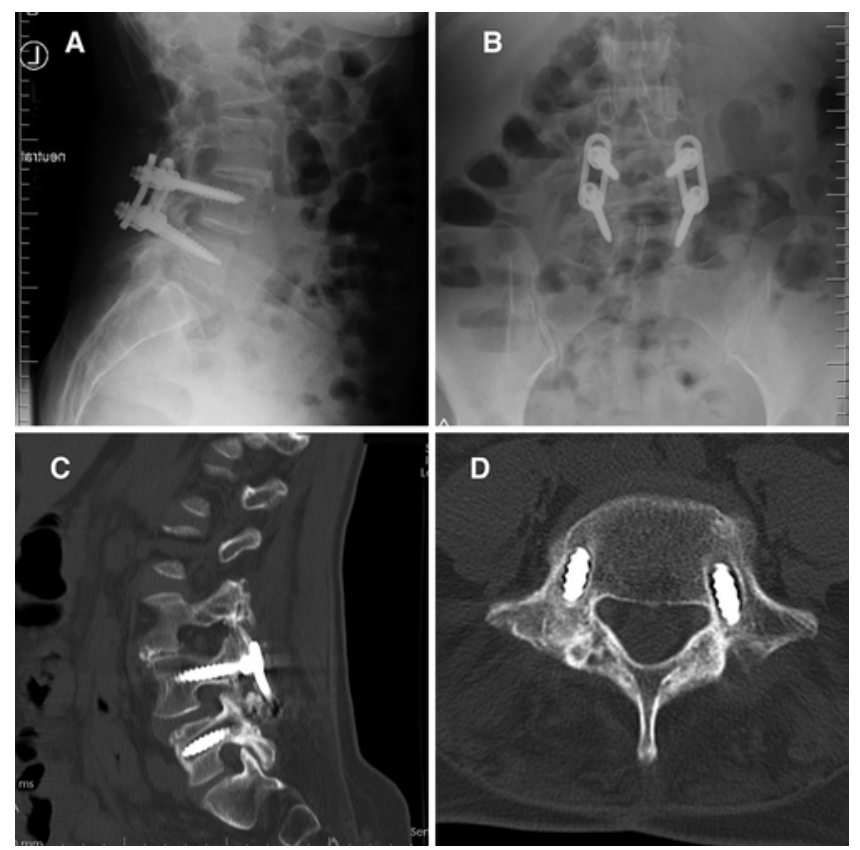

FIG. 9. A and B: Lateral $(A)$ and $A P(B)$ standing radiographs obtained in a 61-year-old woman with failed previous $L 4-5$ fusion. $C$ and D: Notice the radiolucency around bilateral L-5 screws on the preoperative sagittal (C) and axial (D) CT scans. 

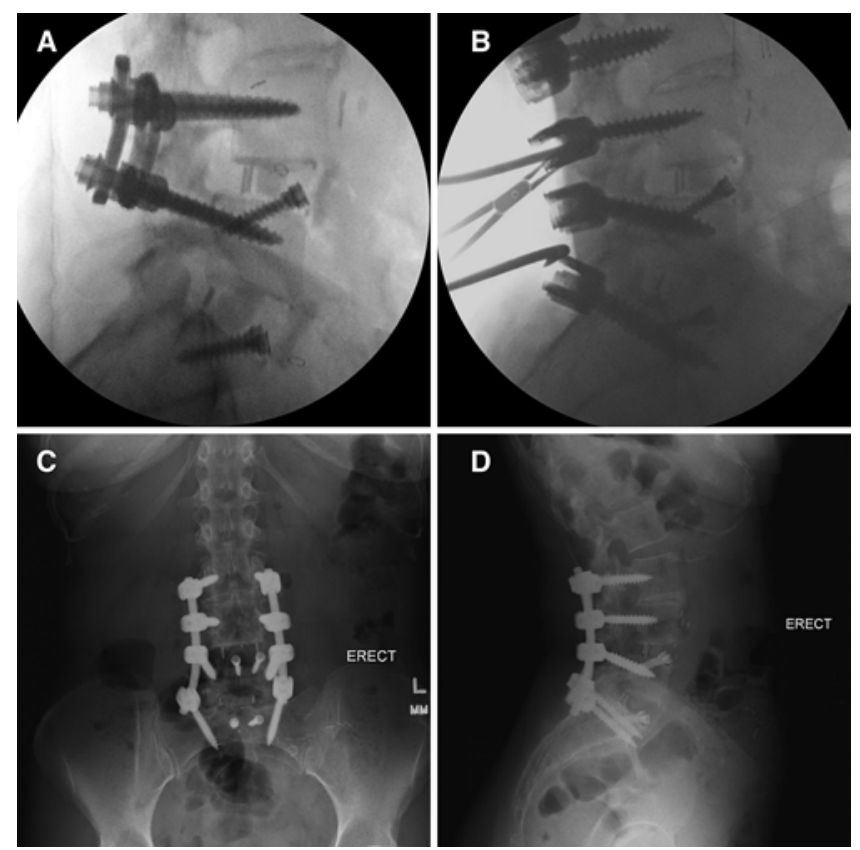

FIG. 10. A: Intraoperative fluoroscopy image obtained after overpowering the L4-5 instrumentation with a $20^{\circ} \mathrm{ALIF}$ cage. In addition, a $30^{\circ}$ cage was also inserted at the L5-S1 level. B: Fluoroscopy image obtained after removal and revision of the posterior instrumentation. C and D: Final postoperative AP (C) and lateral (D) radiographs showing an overall $\mathrm{LL}$ of $70^{\circ}$.

technique. The versatility of our technique was also well demonstrated in patients who had short fusion constructs for spondylolisthesis and persistent neurological symptoms or back pain from previous fusions.

In all patients, careful attention was paid to the presence of pseudarthrosis from previous posterior fusions. In patients with sagittal imbalance with long fusion constructs, CT images of the lumbar spine were reviewed to look for a vacuum sign in the disc spaces, lucency around the screws, and a demarcation line between the facets at fused levels, levels of rod breakages, and so on. Such levels where pseudarthrosis was noted were particularly targeted for the overpowering ALIF procedure, as they were believed to yield the most, thereby offering maximum lordosis correction. This was, however, not proven to be statistically true since the mean increment in SL at levels with pseudarthrosis was not significantly different from those without. However, we found that the overpowering technique was technically easier to perform at levels with pseudarthrosis, and we recommend utilizing these levels to begin with.

TABLE 2. ALIF levels and cages

\begin{tabular}{lccccc}
\hline & \multicolumn{5}{c}{ Levels } \\
\cline { 2 - 6 } Cage & L2-3 & L3-4 & L4-5 & L5-S1 & Total \\
\hline $12^{\circ}$ & 1 & 2 & 1 & 0 & 4 \\
\hline $20^{\circ}$ & 0 & 4 & 3 & 4 & 11 \\
\hline $30^{\circ}$ & 2 & 2 & 6 & 11 & 21 \\
\hline Total & 3 & 8 & 10 & 15 & 36 \\
\hline
\end{tabular}

TABLE 3. Levels with pseudarthrosis

\begin{tabular}{cccc}
\hline & \multicolumn{3}{c}{ No. of Levels w/ Pseudarthrosis } \\
\cline { 2 - 4 } Level & Short Construct & Long Construct & Total \\
\hline L2-3 & 0 & 1 & 1 \\
\hline L3-4 & 1 & 4 & 5 \\
\hline L4-5 & 2 & 2 & 4 \\
\hline L5-S1 & 4 & 4 & 8 \\
\hline Total & 7 & 11 & 18 \\
\hline
\end{tabular}

All patients in our series had previous instrumentation that consisted of titanium/stainless steel rod systems. We believe that this is an important consideration, as some of the correction from the overpowering technique comes from possible flexibility and mild bending of the rods, apart from angular motion and "give" at the crownscrew interface. Constructs of stiffer alloys, such as cobalt chrome, may not be amenable to this technique, and we strongly recommend confirming preoperatively that these constructs were not placed in the previous procedure. The diameters of the screws/rods also have an important bearing on preoperative planning, with smaller diameter screw/ rod systems being likely to yield more with this technique.

The majority of the ALIF cages in our series were implanted at distal lumbar levels: L4-5 $(n=10)$ and L5-S1 $(\mathrm{n}=15)$. This, we believe, is an important advantage, as the ability to correct overall sagittal alignment is maximal with procedures that are done most distally in the lumbar spine. The direct anterior retroperitoneal approach is particularly advantageous for correction at lower lumbar levels, where direct lateral approaches are impossible (L5-S1) or very difficult (L4-5) to perform due to the iliac crest anatomy. Approaching the lumbar spine directly anteriorly (and not laterally or obliquely) makes it easier to achieve SL, as all important steps of the surgery (ALL release, discectomy, distraction with large paddle distractors, release of the PLL, and sequential increments in the hyperlordotic trials) are done in a straight sagittal direction. Another distinct advantage of our technique is the ability to safely use a level where TLIF had been previously performed and fusion is suspect. In our series, we successfully removed 9 TLIF cages ( 3 in the short-construct group and 6 in the long-construct group) from the front and overpowered these levels with hyperlordotic cages to maximize intersegmental lordosis. This would have been otherwise very difficult to achieve safely with other approaches or techniques.

With the described technique, we could reproducibly achieve significant increments in overall LL in our series, with a mean increment of $15.5^{\circ}$ for all cases. The mean increase in LL was more pronounced in the long-construct group than in the short-construct group $\left(22^{\circ}\right.$ vs $\left.7.7^{\circ}\right)$. This can be explained by the redistribution of LL and rebalancing of the SL at mobile lumbar levels proximal to the fused ALIF levels in the short-construct group. Thus, in this group, lumbar levels above the previously fused levels, which were compensating for distal hypolordotic alignment by opening up, fell back into their normal anatomical range of intersegmental lordosis after lordosis restora- 
TABLE 4. Spinopelvic parameters

\begin{tabular}{cccc}
\hline Sagittal Parameter & \multicolumn{1}{c}{ Preop } & Postop & p Value \\
\hline LL $\left(^{\circ}\right)$ & & & \\
\hline Overall & $44.3(15.7 ; 11.3$ to 75.5$)$ & $59.8(7.8 ; 44.9$ to 76$)$ & $<0.05$ \\
\hline Short construct & $55.0(10.7 ; 38$ to 75.5$)$ & $62.7(8.8 ; 52.2$ to 76$)$ & $<0.05$ \\
\hline Long construct & $35.6(13.78 ; 11.3$ to 59.6$)$ & $57.5(6.44 ; 44.9$ to 66.5$)$ & $<0.05$ \\
\hline PT $\left(^{\circ}\right)$ & & & $<0.05$ \\
\hline Overall & $25.2(10.1 ; 9.7$ to 41.3$)$ & $20.4(5.7 ; 8.5$ to 31.5$)$ & $>0.05$ \\
\hline Short construct & $20.5(8.8 ; 9.7$ to 38.1$)$ & $18.2(6.2 ; 8.5$ to 28.6$)$ & $<0.05$ \\
\hline Long construct & $29.0(9.95 ; 15$ to 41.3$)$ & $22.3(4.79 ; 14$ to 31.5$)$ & $<0.05$ \\
\hline SS $\left(^{\circ}\right)$ & & & $>0.05$ \\
\hline Overall & $38.4(6.7 ; 21.8$ to 48.6$)$ & $43.2(8.0 ; 25$ to 56.7$)$ & $<0.05$ \\
\hline Short construct & $38.0(8.0 ; 21.8$ to 45.5$)$ & $40.5(9.7 ; 25$ to 53.4$)$ & $<5.5(5.87 ; 38$ to 56.7$)$ \\
\hline Long construct & $38.8(5.98 ; 30.2$ to 48.6$)$ & & $<0.05$ \\
\hline PI-LL mismatch $\left(^{\circ}\right)$ & & $3.9(11.6 ;-18$ to 28.4$)$ & $<0.05$ \\
\hline Overall & $19.3(20.5 ;-7.8$ to 68.4$)$ & $-3.9(11.0 ;-18$ to 13$)$ & $<0.05$ \\
\hline Short construct & $3.5(9.2 ;-7.8$ to 15.8$)$ & $10.3(7.60 ; 0.8$ to 28.4$)$ & \\
\hline Long construct & $32.2(18.1 ;-1.6$ to 68.4$)$ & & \\
\hline
\end{tabular}

Values are presented as the mean (SD; range).

tion at distal lumbar levels with ALIFs. This effect was obviously not evident in the long-construct group, where the alignment of the lumbar spine was locked into the desired degree of lordosis by the posterior instrumentation, removing any rebalancing effect of the proximal unfused disc levels. Some unseen gain in overall lordosis correction in the long-construct group is also likely achievable because of the posterior releases performed to decompress the dural sac and nerve roots, as well as from overcontouring and in situ bending of the rods during the posterior stage of the procedure. This is hard to isolate and separately account for, since postoperative radiography and measurements (except in 1 case, in which an intermediate erect lateral radiograph was obtained) were done only after the back procedure after the spine was stabilized and instrumented. Also, in some cases in the long-construct group, posterior release was performed at levels other than those overpowered from the front. Nevertheless, good lordosis correction was attained in all patients without the need for pedicle screw osteotomies. In addition to the increase in LL, significant correction in the PI-LL mismatch and SVA was attained in the long-construct group, further demonstrating the utility of our technique in cases of flat-back deformity revision.

A complication rate of $13.3 \%$ for the overpowering ALIF stage, that is, the main corrective procedure, stands out favorably. This rate is more satisfactory than the rates associated with posterior-based TCOs, which have been reported to be as high as $58 \% .^{12}$ Moreover, no neurological deficits, vascular injuries, or retrograde ejaculation in male patients was reported in the postoperative period or follow-up duration.

TABLE 5. SL correction

\begin{tabular}{|c|c|c|c|c|}
\hline \multirow[b]{2}{*}{ Cage Type } & \multicolumn{3}{|c|}{ SL Correction } & \multirow[b]{2}{*}{$\mathrm{p}$ Value } \\
\hline & Preop & Postop & $\Delta S L$ & \\
\hline $12^{\circ}(n=4)$ & $6.9(2.1 ; 5.0$ to 9.5$)$ & $13.1(1.6 ; 11.6$ to 15.4$)$ & $6.1(3.2 ; 3.5$ to 10.1$)$ & $<0.05$ \\
\hline $20^{\circ}(n=11)$ & $6.4(5.8 ;-4.5$ to 15$)$ & $19.0(3.0 ; 15.7$ to 24.2$)$ & $12.5(5.5 ; 5.4$ to 20.5$)$ & $<0.05$ \\
\hline $30^{\circ}(n=21)$ & $4.6(5.0 ;-3$ to 15.1$)$ & $22.4(4.8 ; 12.7$ to 28.2$)$ & 17.7 (5.2; 9.6 to 27$)$ & $<0.05$ \\
\hline
\end{tabular}

$\Delta S L=$ mean increase in $\mathrm{SL}$.

Values are presented as the mean (SD; range).

TABLE 6. Segmental lordosis (pseudarthrosis vs nonpseudarthrosis levels)

\begin{tabular}{|c|c|c|c|}
\hline \multirow[b]{2}{*}{ Pseudarthrosis } & \multicolumn{2}{|c|}{ SL Correction } & \multirow[b]{2}{*}{$\mathrm{p}$ Value } \\
\hline & Preop & Postop & \\
\hline Yes & $5.7(5.1 ;-2.2$ to 15.1$)$ & $19.9(5.6 ; 11.6$ to 28.2$)$ & $<0.05$ \\
\hline No & $5.16(5.12 ;-4.5$ to 15$)$ & $20.85(4.52 ; 13$ to 28$)$ & $<0.05$ \\
\hline
\end{tabular}

Values are presented as the mean (SD; range). 
TABLE 7. Complications

\begin{tabular}{lc}
\hline \multicolumn{1}{c}{ Complications } & $\begin{array}{c}\text { No. of } \\
\text { Cases (\%) }\end{array}$ \\
\hline Related to anterior surgery & \\
\hline$\quad$ Fluid collection/seroma in anterior abdominal wall & $1(5)$ \\
\hline$\quad$ Endplate impaction/collapse & $3(8.3)^{*}$ \\
\hline Related to posterior surgery/overall & $1(5)$ \\
\hline$\quad$ Wound dehiscence/infection & $1(5)$ \\
\hline$\quad$ Proximal junctional kyphosis/failure & $1(5)$ \\
\hline$\quad$ Pulmonary embolism/DVT & \\
\hline DVT = deep venous thrombosis. & \\
$*$ Percentage per level of ALIF.
\end{tabular}

To conclude, several factors led to the successful implementation of the overpowering technique in our small series of patients. Surgeon experience with rigorous anterior disc preparation with ALL and PLL removal/resection and careful distraction on endplates to avoid endplate fracture/ comminution was paramount in performing this procedure safely. Similarly, excluding osteoporotic patients and treating levels with pseudarthrosis and frank hardware failure of the posterior elements allowed for easier anterior distraction. In addition, instrumentation characteristics like non-cobalt chrome rods allowed more flexibility with anterior correction. Finally, and most importantly, the postoperative SL was a result of both the anterior cage and the posterior release and compression. Thus, the anterior strut did not do the overpowering alone. Rather, an oversized/hyperlordotic cage was placed in the highest level possible without causing endplate fracture, and then the posterior elements were adjusted to improve lordosis.

Limitations of our study include the small number of patients in the series as well as a heterogeneous mix of cases, making generalization difficult. An 11.5-month follow-up period provides good early radiographic data for correction and maintenance of alignment; however, longer follow-up is needed to confirm lasting results. Our series also does not include a study of fusion rates and clinical outcomes, as the primary goal was to evaluate feasibility and magnitude of alignment correction from the front. It is also a retrospective single-surgeon series, and the results obtained by the senior author, who has significant experience with this technique, may not be uniformly reproducible because of the associated learning curve.

\section{Conclusions}

Overpowering posterior lumbar instrumentation and/ or fusion with hyperlordotic ALIF spacers can be safely and effectively performed and may prove to be a useful addition to the techniques for lumbar sagittal plane reconstruction in revision scenarios. The degree of SL correction can be predicted to be half of the lordotic angle of the cage used to overpower the posteriorly fused level. In addition to meticulous surgical technique, patient selection and a detailed analysis of the previous instrumentation and fusion are crucial for optimizing gains and avoiding complications with this technique. The technique is particularly well suited for application at lumbar levels with pseudarthrosis from previous posterior instrumentation and fusion. Distinct advantages of the technique include the ability of lordosis recreation at the lower lumbar levels, previously fused posteriorly in a suboptimal alignment, less blood loss, and lower rates of neurological complications compared with posterior-based TCOs.

\section{References}

1. Akbarnia BA, Mundis GM Jr, Moazzaz P, Kabirian N, Bagheri R, Eastlack RK, et al: Anterior column realignment (ACR) for focal kyphotic spinal deformity using a lateral transpsoas approach and ALL release. J Spinal Disord Tech 27:29-39, 2014

2. Arlet V: Spinal osteotomy in the presence of massive lumbar epidural scarring. Eur Spine J 24 (Suppl 1):S93-S106, 2015

3. Auerbach JD, Lenke LG, Bridwell KH, Sehn JK, Milby $\mathrm{AH}$, Bumpass D, et al: Major complications and comparison between 3-column osteotomy techniques in 105 consecutive spinal deformity procedures. Spine (Phila Pa 1976) 37:1198-1210, 2012

4. Berjano P, Cecchinato R, Sinigaglia A, Damilano M, Ismael MF, Martini C, et al: Anterior column realignment from a lateral approach for the treatment of severe sagittal imbalance: a retrospective radiographic study. Eur Spine J 24 (Suppl 3):433-438, 2015

5. Bianco K, Norton R, Schwab F, Smith JS, Klineberg E, Obeid $\mathrm{I}$, et al: Complications and intercenter variability of threecolumn osteotomies for spinal deformity surgery: a retrospective review of 423 patients. Neurosurg Focus 36(5):E18, 2014

6. Buchowski JM, Bridwell KH, Lenke LG, Kuhns CA, Lehman RA Jr, Kim YJ, et al: Neurologic complications of lumbar pedicle subtraction osteotomy: a 10-year assessment. Spine (Phila Pa 1976) 32:2245-2252, 2007

7. Deukmedjian AR, Dakwar E, Ahmadian A, Smith DA, Uribe JS: Early outcomes of minimally invasive anterior longitudinal ligament release for correction of sagittal imbalance in patients with adult spinal deformity. Sci World J 2012:789698, 2012

8. Hassanzadeh H, Jain A, El Dafrawy MH, Ain MC, Mesfin A, Skolasky RL, et al: Three-column osteotomies in the treatment of spinal deformity in adult patients 60 years old and older: outcome and complications. Spine (Phila Pa 1976) 38:726-731, 2013

9. Manwaring JC, Bach K, Ahmadian AA, Deukmedjian AR, Smith DA, Uribe JS: Management of sagittal balance in adult spinal deformity with minimally invasive anterolateral lumbar interbody fusion: a preliminary radiographic study. J Neurosurg Spine 20:515-522, 2014

10. Mummaneni PV, Shaffrey CI, Lenke LG, Park P, Wang MY, La Marca F, et al: The minimally invasive spinal deformity surgery algorithm: a reproducible rational framework for decision making in minimally invasive spinal deformity surgery. Neurosurg Focus 36(5):E6, 2014

11. Murray G, Beckman J, Bach K, Smith DA, Dakwar E, Uribe JS: Complications and neurological deficits following minimally invasive anterior column release for adult spinal deformity: a retrospective study. Eur Spine J 24 (Suppl 3):397-404, 2015

12. Norton RP, Bianco K, Lafage V, Schwab FJ: Complications and intercenter variability of three-column resection osteotomies for spinal deformity surgery: a retrospective review of 423 patients. Evid Based Spine Care J 4:157-159, 2013

13. Rajaee SS, Kanim LEA, Bae HW: National trends in revision spinal fusion in the USA: patient characteristics and complications. Bone Joint J 96-B:807-816, 2014

14. Rothenfluh DA, Mueller DA, Rothenfluh E, Min K: Pelvic 
incidence-lumbar lordosis mismatch predisposes to adjacent segment disease after lumbar spinal fusion. Eur Spine J 24:1251-1258, 2015

15. Saigal R, Mundis GM Jr, Eastlack R, Uribe JS, Phillips FM, Akbarnia BA: Anterior column realignment (ACR) in adult sagittal deformity correction: technique and review of the literature. Spine (Phila Pa 1976) 41 (Suppl 8): S66-S73, 2016

16. Saville PA, Kadam AB, Smith HE, Arlet V: Anterior hyperlordotic cages: early experience and radiographic results. J Neurosurg Spine 25:713-719, 2016

17. Schwab F, Lafage V, Patel A, Farcy JP: Sagittal plane considerations and the pelvis in the adult patient. Spine (Phila Pa 1976) 34:1828-1833, 2009

18. Schwab F, Patel A, Ungar B, Farcy JP, Lafage V: Adult spinal deformity-postoperative standing imbalance: how much can you tolerate? An overview of key parameters in assessing alignment and planning corrective surgery. Spine (Phila Pa 1976) 35:2224-2231, 2010

19. Turner JD, Akbarnia BA, Eastlack RK, Bagheri R, Nguyen S, Pimenta L, et al: Radiographic outcomes of anterior column realignment for adult sagittal plane deformity: a multicenter analysis. Eur Spine J 24 (Suppl 3):427-432, 2015

20. Umehara S, Zindrick MR, Patwardhan AG, Havey RM, Vrbos LA, Knight GW, et al: The biomechanical effect of postoperative hypolordosis in instrumented lumbar fusion on instrumented and adjacent spinal segments. Spine (Phila Pa 1976) 25:1617-1624, 2000

\section{Disclosures}

Dr. Arlet reports that he is a consultant for NuVasive, DePuy, and Zimmer; patent holder with Medtronic; and is on the faculty of AOSpine.

\section{Author Contributions}

Conception and design: Arlet. Acquisition of data: Kadam, Wigner, Saville. Analysis and interpretation of data: Kadam, Wigner, Saville. Drafting the article: Kadam, Wigner. Critically revising the article: Arlet, Kadam, Saville. Reviewed submitted version of manuscript: Arlet, Saville. Approved the final version of the manuscript on behalf of all authors: Arlet. Statistical analysis: Kadam, Saville. Administrative/technical/material support: Arlet. Study supervision: Arlet.

\section{Correspondence}

Vincent Arlet, Department of Orthopaedics, University of Pennsylvania, 235 S 8th St., Washington West Bldg., Philadelphia, PA 19106. email: arletvincent@gmail.com. 